\title{
TECNOLOGIA LEVE NO PÓS-PARTO: MATERIAL EDUCATIVO COMO INSTRUMENTO DA EXTENSÃO UNIVERSITÁRIA
}

\section{POSTPARTUM LIGHT TECHNOLOGY: EDUCATIONAL MATERIAL AS AN INSTRUMENT OF UNIVERSITY OUTREACH}

\begin{abstract}
:
Health education is very important, in which nursing has a broad action towards the community, clarifying, guiding and educating for health. This study aimed to devise educational material in a teaching maternity in Campos Gerais, as well as applying and evaluating such material, about postpartum and breastfeeding with puerperal women and specialists in the primary, secondary and tertiary level according to Pasquali's criteria. This is a descriptive study through the action of an outreach project in contact with the community. The collection was carried out with ten puerperal women assisted by the project and ten specialists in the area of women's health/Obstetrics. The educational material on Postpartum was created to solve the doubts of the participants in interaction with the team of the Nursing Consultation Project in Prenatal and Postpartum outreach project. The results showed that all the criteria evaluated by the specialists and puerperal women exceeded the target average of $50 \%$, reaching between 80 and $100 \%$ total agreement, leading to the conclusion that the educational material facilitated the puerperal women's learning, contributing to maternal autonomy.
\end{abstract}

Keywords: Nursing consultation; Health education; Postpartum period; Breastfeeding.

\footnotetext{
*Aluna de Graduação da Universidade Estadual de Ponta Grossa (UEPG), Ponta Grossa - PR, Brasil. E-mail: even_m@outlook.com

${ }^{* *}$ Professora da Universidade Estadual de Ponta Grossa (UEPG), Ponta Grossa - PR, Brasil. E-mail: anapxr@hotmail.com
} 


\section{Introdução}

Gestar uma vida é um momento singular para a mulher, ocorrem mudanças físicas, emocionais e hormonais que surgem paulatinamente. Diante disso, as alterações acometem a postura anatômica, como também o sistema digestório, urinário, respiratório e tegumentar (REGRA; SALERNO; FERNANDES, 2017). Ao final da gestação, a mulher vivencia o momento do parto, ou seja, o nascimento de seu filho(a), em que a sensação de dor e desconforto se entrelaça com a plenitude do amor incondicional, traduzida na alegria de ser mãe.

Logo após o parto e dequitação placentária, uma nova fase se inicia, chamada de Pós-Parto, Resguardo, Dieta ou Puerpério, que é um momento delicado para a mulher, seu bebê e sua família, durando em torno de seis semanas, respeitando a condição materna. A variação ocorre de acordo com as mudanças anatômicas e físiológicas de cada mulher, além de certos fatores como a ordem psicossocial, que envolve a maternidade, a sexualidade, a autoestima, a reorganização da vida pessoal e familiar, tudo influenciando de maneira significava nesta fase (BRASIL, 2016).

Segundo o Ministério da Saúde (BRASIL, 2016), o puerpério pode ser dividido em imediato $\left(1^{\circ}\right.$ ao $10^{\circ}$ dia após o parto), tardio (do $11^{\circ}$ ao $45^{\circ}$ dia) e remoto (após o $45^{\circ}$ dia). Vale destacar que o ciclo gravídico-puerperal proporciona vivências singulares às mulheres, e para que esse ciclo ocorra de forma adequada, ressalta-se a importância dos profissionais de saúde envolvidos em todo o processo, destacando-se o enfermeiro. Sua assistência deve abranger um conjunto de ações com vistas à promoção da saúde, do acolhimento, vínculo, e assim desenvolver a autonomia da mulher para o seu autocuidado e com o(a) filho(a) (FIGUEIREDO et. al., 2018; MARINHO; ANDRADE; ABRÃO, 2015).

Diante disso, salienta-se a importância da educação em saúde no decorrer de todo o ciclo gravídico-puerperal, em que a enfermagem apresenta uma ampla ação educativa, sendo provedora do processo de aprendizado com as mulheres de forma efetiva, com base no conhecimento científico, visando a autonomia materna no cuidado consigo e com seu bebê (FIGUEIREDO et al., 2018; MENDES et al., 2016).

No Pós-Parto, a assistência puerperal requer cuidados atentos dos profissionais, acompanhando e entendendo o que se passa com cada puérpera e a família, fornecendo suporte de forma educativa e assistencial, podendo sanar as dúvidas ou dificuldades na fase vivida. Cabe ressaltar que o enfermeiro precisa observar e identificar aspectos relevantes do cuidar, para, assim, promover a educação em saúde esclarecedora (PRIGOL; BARUFFI, 2017).

Neste contexto, a mulher necessita ser atendida em sua totalidade, de maneira integral, considerando o seu contexto sociocultural e familiar. Os profissionais de saúde precisam estar preparados para perceber as necessidades apresentadas por essas mulheres e assim promover a educação em saúde (PRIGOL; BARUFFI, 2017).

Desta forma, para que haja uma boa relação profissional e puérpera, é preciso ter uma boa comunicação e relações interpessoais, que são a base para o cuidado em saúde (ABREU; AMENDOLA; TROVO, 2017). Nesta perspectiva, o cuidado em saúde pode ocorrer por meio de tecnologias leves, o que constitui as relações entre os sujeitos da ação, implementando o cuidado, vínculo, gestão de serviços e acolhimento (SABINO et al., 2016). Este estudo se apropriará da definição de tecnologia leve, segundo Merhy, centrado no campo das relações que envolvem acolhimento e vínculo entre os profissionais e usuários (MERHY, 2003, apud OLIVEIRA; SUTO; SILVA, 2016, p. 614).

Sendo assim, a tecnologia leve se apresenta como uma ferramenta importante na atuação direta com a mulher que vivencia o ciclo gravídico-puerperal, a partir do vínculo e a apropriação de materiais educativos que subsidiam a relação. Para isso, é necessário que o material seja estruturado a partir de dados científicos e evidência, numa linguagem clara e objetiva, para acontecer o aprendizado posterior. 
Vale ressaltar que os materiais educativos fazem com que o ensino-aprendizagem se torne efetivo, com interações mediadas por um locutor (enfermeiro), usuária (mulher) e família (FARIAS et al., 2018). No entanto, é necessário que seja atrativo, acessível e claro, significativo e adepto à realidade do leitor, não devendo somente transmitir informações, mas, sim, estimular a reflexão e facilitar a instrumentalização para o cuidar (DALMOLIN et al., 2016).

Diante do exposto, a extensão universitária pode contribuir com a necessidade da comunidade, aqui destacando as mulheres no pós-parto, a partir de projetos e programas que subsidiam o aprendizado, visando facilitar os cuidados diários. Foi assim que o Projeto Consulta de Enfermagem no Pré-Natal e Pós-Parto (CEPP) iniciou suas ações de extensão, mediante uma necessidade local. Desta forma, extensão universitária é a articulação do conhecimento científico, advindo do ensino e da pesquisa, com as necessidades da comunidade onde a universidade se insere, interagindo e transformando a realidade social (NASCIMENTO et al., 2015).

Portanto, este estudo objetivou estruturar um material educativo sobre pós-parto e aleitamento materno em uma maternidade de ensino nos Campos Gerais, bem como aplicá-lo e avaliá-lo junto a puérperas e especialistas em âmbito primário, secundário e terciário, segundo critérios de Pasquali.

\section{Metodologia}

O estudo é descritivo, com caráter dialético, uma vez que é uma atividade que busca soluções para problemas do cotidiano, descobrindo conhecimento novo, compreensão e, se possível, a intervenção na realidade (MATOS et al., 2019).

O material educativo impresso é um livreto que contém informações sobre pós-parto e aleitamento Materno, baseado em evidências advindas do Ministério da Saúde, o qual foi elaborado com o intuito de sanar as dúvidas das puérperas. A atuação educativa do projeto CEPP aborda assuntos como: O que é puerpério? É normal sentir cólica? O que é o sangramento puerperal: dieta, mitos e verdades. Manobra de Heimlich. Aleitamento Materno Composição do leite materno. Problemas para amamentar e quando procurar ajuda médica.

Vale ressaltar que o projeto CEPP desenvolve ações extensionistas desde 2006 com mulheres no pós-parto, a partir da educação em saúde no pós-parto, por meio de consulta de enfermagem, com acadêmicos do curso de Bacharelado em Enfermagem do $4^{\circ}$ e $5^{\circ}$ anos, bem como um jogo de memória, instrumento lúdico usado para esclarecer as dúvidas das puérperas e familiares. O material educativo será usado para complementar e embasar as ações de extensão propostas pelo projeto, com informações e figuras ilustrativas, visando contribuir com o conhecimento adquirido pela comunidade assistida.

Sendo assim, a coleta ocorreu com 10 puérperas atendidas pelo projeto CEPP e 10 especialistas na área da saúde da mulher e/ou obstetrícia, que aconteceu nos meses de março e abril de 2019, em uma Maternidade referência ao risco habitual e intermediário na região dos Campos Gerais.

Foram utilizados 2 questionários estruturados, visando a avaliação do material educativo construído mediante os critérios estabelecidos por Pasquali (PASQUALI, 2009; CRESTANI; MORAES; SOUZA, 2017), que descreve a teoria da elaboração de instrumento de medida de fenômenos subjetivos. Destaca-se que, no Instrumento, há afirmativas condizentes aos critérios: Interatividade, Objetivos, Relevância e Eficácia, Clareza, Estrutura e Apresentação.

Ainda, a partir das afirmações, apropriou-se da escala de Likert, para fins de análise e mensuração dos critérios, tendo como média alvo $\geq 50 \%$ de acertos, e como legenda: Concordo Totalmente, Concordo Parcialmente e Discordo. 
Este estudo teve como critérios de inclusão mulheres internadas na maternidade escola vivenciando o puerpério nos meses de coleta, bem como as especialistas, enfermeiras da área da saúde da mulher e/ou obstetrícia atuantes na maternidade de referência. Por sua vez, os critérios de exclusão foram as demais mulheres internadas e os demais especialistas de outras áreas.

A análise dos dados aconteceu mediante estatística descritiva, a partir de frequência simples/percentual, tendo como base a escala de Likert e média alvo estimada de $50 \%$. Todos os sujeitos da amostra foram convidados e assinaram o Termo de Consentimento Livre e Esclarecido, de acordo com a Resolução 466 de 2012. O estudo tem parecer do Comitê de Ética em Pesquisa pela Universidade Estadual de Ponta Grossa, sob Parecer 3.234.262 de 29 de março de 2019.

\section{Resultados}

Iniciar-se-á apresentando uma breve caracterização da amostra do estudo, começando pelas especialistas, totalizando $100 \%(\mathrm{n}=10)$ do sexo feminino, profissão enfermeiras, sendo: $10 \%$ do âmbito hospitalar $(\mathrm{n}=1), 50 \%$ do ponto de apoio da rede de saúde $(n=5)$ e $40 \%$ da atenção básica $(n=4)$. Quanto à faixa etária, $40 \%$ apresentaram idade entre 30 e 39 anos e 30\% apresentaram idade entre 40 e 49 anos. Quanto ao tempo de formação, $\geq 10$ anos com $60 \%(n=6)$, e atuação como enfermeira obstetra, $\geq 2$ anos num total de $70 \%(n=7)$ e os outros $30 \%(n=3) \geq 10$ anos. Quanto ao período de maior atuação no ciclo gravídico-puerperal, destacou o parto e pós-parto, com $90 \%(n=9)$.

Prosseguindo, $80 \%(\mathrm{n}=8)$ tinham idade entre 20 e 29 anos, ao passo que $80 \%$ $(n=8)$ tinham estado civil casadas/relação estável. Já os anos de escolaridade, $60 \%(n=6)$ tinham de 6 a 9 anos de estudo, com destaque para o Ensino Fundamental completo com $40 \%(n=4)$ e $30 \%(n=3)$ Ensino Médio completo. Por sua vez, $100 \%(n=10)$ não trabalhavam fora, disseram ser do lar; e 100\% $(n=10)$ tinham renda familiar entre 1 a 2 salários mínimos e $70 \%(\mathrm{n}=7)$ tinham residência própria.

Após a estruturação do material educativo, este foi encaminhado às especialistas e puérperas para apropriação e avaliação, em que se obtiveram os dados conforme critérios de Pasquali, segundo Tabela 1.

Tabela 1 - Avaliação do Material Educativo segundo critérios de Pasquali (2010) e Likert - Especialistas. Cepp 2019.

\begin{tabular}{|c|c|c|c|c|c|c|c|}
\hline \multirow{2}{*}{ Critérios } & \multirow{2}{*}{ Questões Afirmativas } & \multicolumn{2}{|c|}{ CT } & \multicolumn{2}{|c|}{$\mathrm{CP}$} & \multicolumn{2}{|c|}{$\mathbf{D}$} \\
\hline & & $\mathbf{N}$ & $\%$ & $\mathbf{N}$ & $\%$ & $\mathbf{N}$ & $\%$ \\
\hline \multirow[t]{4}{*}{ Interatividade } & 1.0 conteúdo está adequado. & 9 & 90 & 1 & 10 & 0 & 0 \\
\hline & 2. Favorece o processo educativo & 10 & 100 & 0 & 0 & 0 & 0 \\
\hline & 3. Possibilita entendimento do conteúdo. & 8 & 80 & 2 & 20 & 0 & 0 \\
\hline & 4. Fornece autonomia ao usuário em relação à sua apresentação. & 9 & 90 & 1 & 10 & 0 & 0 \\
\hline \multirow[t]{4}{*}{ Objetivos } & 5. Estimula aprendizagem sobre conteúdo abordado. & 10 & 100 & 0 & 0 & 0 & 0 \\
\hline & 6. Estimula aprendizagem de novos conceitos. & 90 & 90 & 1 & 10 & 0 & 0 \\
\hline & 7. Permite buscar informações sem dificuldades. & 9 & 90 & 1 & 10 & 0 & 0 \\
\hline & 8. Possui estratégia de apresentação atrativa. & 9 & 90 & 1 & 10 & 0 & 0 \\
\hline \multirow[t]{4}{*}{ Relevância e Eficácia } & 9. Disponibiliza fácil utilização. & 9 & 90 & 1 & 10 & 0 & 0 \\
\hline & 10. Desperta interesse para utilizá-lo. & 10 & 100 & 0 & 0 & 0 & 0 \\
\hline & 11. Estimula mudança de comportamento/autonomia. & 8 & 80 & 2 & 20 & 0 & 0 \\
\hline & 12. Reproduz o conteúdo abordado em diferentes fases do pós-parto. & 9 & 100 & 1 & 10 & 0 & 0 \\
\hline \multirow[t]{2}{*}{ Clareza } & 13. Apresenta informações de modo simples. & 9 & 90 & 1 & 10 & 0 & 0 \\
\hline & 14. Permite refletir sobre o conteúdo apresentado. & 10 & 100 & 0 & 0 & 0 & 0 \\
\hline
\end{tabular}

Fonte: Dados da pesquisadora mediante banco de dados do projeto CEPP. Legenda: CP: Concordo Parcialmente, CT: Concordo Totalmente e D: Discordo. 
Ressalta-se que todos os critérios superaram a média-alvo de $50 \%$. Nos critérios Interatividade e Relevância/Eficácia, destacam-se os que alcançaram $80 \%$ de respostas CT, "Possibilita entendimento do conteúdo" e "Estimula mudança de comportamento/ autonomia materna", tendo os demais $20 \%$ de especialistas avaliado com CP tais critérios frente ao Material Educativo. Isso indica que, para a visão das enfermeiras especialistas em Saúde da Mulher/Obstetrícia, o material educativo conseguiu atingir seus objetivos e poderá contribuir com o aprendizado de puérperas frente ao período vivido de maneira clara, numa linguagem acessível.

No olhar das especialistas, das 14 afirmações expostas, todas obtiveram mínimo de $80 \%$ e máximo de $100 \%$ de concordância total (CT). Isso remete a uma avaliação positiva do material educativo, que apresenta novos conceitos a serem apreendidos, de modo que as puérperas conseguirão buscar informações sem dificuldades, além de contar com uma apresentação atrativa. O material foi criado para promover a autonomia das puérperas no processo de conhecimento frente à involução do próprio corpo no pós-parto e a manutenção da saúde. Nesta perspectiva, o material pode auxiliar no processo de construção do conhecimento (PEUKER et al., 2017).

Assim sendo, com a elaboração do material educativo, que é uma tecnologia impressa, apesar de simples, é possível alcançar maior número de pessoas, pois o desenvolvimento e a implementação de tecnologias educativas favorecem mudanças comportamentais, e aqui podem tornar as puérperas confiantes para a realização do autocuidado e os cuidados com o recém-nascido e frente ao Aleitamento Materno (LIMA et al., 2018).

Vale ressaltar que o material impresso não visa substituir a orientação do profissional da saúde, mas sim atrair a comunidade, aqui, as puérperas, na busca do conhecimento. Assim, poderá contribuir no processo de construção do saber, como também chamando a atenção para o assunto, com reflexões a respeito da saúde materna e neonatal (PEUKER et al., 2017).

Desta forma, conforme a Tabela 1, pôde-se evidenciar a importância da extensão universitária atuando diretamente com a comunidade, a partir do conhecimento científico por meio do material educativo, que se mostrou eficiente para contribuir no aprendizado da comunidade assistida, segundo a visão dos especialistas.

Tabela 2 - Avaliação do Material Educativo segundo critérios de Pasquali (2010) Puérperas. Cepp 2019.

\begin{tabular}{|c|c|c|c|c|c|c|c|}
\hline \multirow{2}{*}{ Critérios } & \multirow{2}{*}{ Questões Afirmativas } & \multicolumn{2}{|c|}{ CT } & \multicolumn{2}{|c|}{$\mathbf{C P}$} & \multicolumn{2}{|c|}{ D } \\
\hline & & $\mathbf{N}$ & $\%$ & $\mathbf{N}$ & $\%$ & $\mathbf{N}$ & $\%$ \\
\hline \multirow[t]{3}{*}{ Objetivos } & 1. O conteúdo abordado tem relação no seu dia a dia. & 9 & 90 & 1 & 10 & 0 & 0 \\
\hline & 2. Esclarece as dúvidas sobre o conteúdo abordado. & 10 & 100 & 0 & 0 & 0 & 0 \\
\hline & 3. Estimula aprendizagem sobre o conteúdo abordado. & 10 & 100 & 0 & 0 & 0 & 0 \\
\hline \multirow[t]{3}{*}{ Clareza } & $\begin{array}{l}\text { 4. Apresenta informações necessárias para compreensão do tema } \\
\text { pós-parto e aleitamento. }\end{array}$ & 9 & 90 & 1 & 10 & 0 & 0 \\
\hline & $\begin{array}{l}\text { 5. As ilustrações contidas no panfleto educativo são de fácil } \\
\text { entendimento e possuem uma boa coloração. }\end{array}$ & 9 & 90 & 1 & 10 & 0 & 0 \\
\hline & 6. Apresenta informações de modo simples. & 8 & 80 & 2 & 20 & 0 & 0 \\
\hline \multirow{2}{*}{$\begin{array}{l}\text { Estrutura e } \\
\text { apresentação }\end{array}$} & 7. Apresenta conteúdo de forma organizada. & 10 & 100 & 0 & 0 & 0 & 0 \\
\hline & 8. Possui estratégia de apresentação atrativa. & 10 & 100 & 0 & 0 & 0 & 0 \\
\hline \multirow[t]{2}{*}{ Interatividade } & 9. Oferece interação, envolvimento ativo no processo educativo. & 10 & 100 & 0 & 0 & 0 & 0 \\
\hline & 10. Fornece autonomia materna em relação ao seu manuseio. & 10 & 100 & 0 & 0 & 0 & 0 \\
\hline
\end{tabular}

Fonte: Dados da pesquisadora mediante banco de dados do projeto CEPP. Legenda: CP: Concordo Parcialmente, CT: Concordo Totalmente e D: Discordo. 
Com relação ao olhar das puérperas, conforme Tabela 2, todos os critérios superaram a média-alvo de 50\%, ficando entre 80 a 100\% CT. Somente a afirmativa "Apresenta informações de modo simples" atingiu $80 \%$ de CT e demais $20 \%$ com CP. Sendo assim, com um índice de concordância total satisfatório, pode-se indicar que o material educativo proposto é uma ferramenta eficaz no processo ensino (enfermeiro) e aprendizagem (puérperas), a partir da educação em saúde desenvolvida sobre o pós-parto e aleitamento materno.

Para tanto, existem 12 critérios que julgam os itens de um instrumento, que são relacionados com o referencial metodológico de Pasquali, que irá dar subsídios para a avaliação de conteúdo, porque faz com que avaliem propriedade psicométricas do instrumento e indiquem se os itens são compreensíveis à população-alvo (PASQUALI, 2009, 2010 apud CRESTANI; MORAES; SOUZA, 2017, p. 2).

Como reflexão, o conhecimento adquirido pelas puérperas a partir da educação em saúde com o uso de instrumentos facilitadores, aqui o Material Educativo, pode sugerir uma aprendizagem contemplada com os aspectos plurais da formação humana, com a participação ativa, interação, capacidade de autorreflexão e conquista do conhecimento (MATOS et al., 2019).

Em face disso, a educação em saúde é considerada principalmente como autogerenciamento, com vistas à uma melhor condição de saúde e de qualidade de vida, principalmente em período tão delicado para a mulher. Observa-se que a intervenção educativa deve ser iniciada precocemente com a mulher, a qual pode assimilar o cuidado por meio do material impresso. É preciso a presença de profissionais juntamente com as puérperas para explicar o material e o conteúdo exposto, a fim de que elas não sejam sobrecarregada de informações, e não fiquem sem entender o que significam (NASCIMENTO et al., 2015).

Assim se percebeu a necessidade do desenvolvimento de um material que trouxesse informações relevantes para o conhecimento da mulher sobre as alterações que estão ocorrendo em seu corpo. E, com isso, que elas questionem suas dúvidas e troquem experiências para a resolução de problemas que favoreçam o momento vivido. Ressalta-se que o material impresso jamais irá substituir a orientação de um profissional da saúde, mas pode promover a atração da população feminina que vive o puerpério. Além disso, o assunto transmitido é apenas aplicado no cotidiano das puérperas (PEUKER et. al., 2017).

Destaca-se a importância da utilização do material educativo como meio de ensino-aprendizagem, pois gera um ambiente facilitador para a transmissão do conhecimento, que passa a ser proporcionado tanto para o profissional responsável pela educação em saúde quanto para a mulher que está no período pós-parto. Isso gera uma adesão satisfatória dos conhecimentos transmitidos, com aplicação prática na rotina social e familiar das puérperas (MATOS et al., 2019).

Enfatiza-se o uso de imagens nos materiais educativos, pois além do conteúdo, sua visualização contribui para a inteligibilidade de diversos textos científicos, e que tais imagens têm um papel importante na constituição de ideais e na sua contextualização. Além disso, uma ilustração pode ser autoexplicativa, pois supera a barreira da linguagem (D’AVILA; PUGGINA; FERNANDES, 2018).

Com isso, a enfermagem deve desenvolver ações educativas que não sejam apenas no repasse de informações, mas que estejam envolvidas na demanda das puérperas e à sua realidade sociocultural. Portanto, é preciso associar ao seu cuidado a educação em saúde, com a relação horizontalizada com os sujeitos (DODOU et al., 2017).

$\mathrm{Na}$ visão da puérperas, no decorrer de sua internação no período pós-parto, implementar a educação em saúde utilizando instrumentos facilitadores do aprendizado, aqui o Material Educativo, pode contribuir na autonomia materna frente aos cuidados consigo e com o bebê. Assim, as ações de extensão universitária desenvolvidas pelo 
projeto CEPP com a comunidade de puérperas na rede municipal de saúde tem sua importância, pois apresenta uma função social, respeitando culturalmente a população assistida e promovendo o desenvolvimento social.

Por fim, acredita-se que a elaboração do material educativo na forma impressa pode ser um facilitador do ensino-aprendizagem com a comunidade, na qual se deve considerar a cultura, bem como utilizar uma linguagem acessível, pois, assim, tanto os emissores (enfermeiros) como os receptores (puérperas) poderão juntos construir um conhecimento, com troca de saberes e um interesse em comum, o aprendizado (NASCIMENTO et al., 2015).

\section{Conclusão}

Este estudo estruturou um material educativo impresso, que foi planejado, confeccionado e avaliado por puérperas e especialistas. Observou-se que o material facilitou o aprendizado das puérperas, público-alvo do projeto CEPP. Assim, pôde-se evidenciar que houve interesse pelo material educativo oferecido, pois continha uma linguagem clara, atrativa quanto às cores e ilustrações, minimizando as dúvidas sobre o pós-parto e o aleitamento materno.

É importante destacar que o ensino-aprendizagem é um processo na prática da educação em saúde, numa relação entre enfermeiro/puérpera, ou seja, meio facilitador do conhecimento na prática assistencial, refletindo no contexto social e familiar da comunidade assistida.

Vale ressaltar, ainda, a importância da extensão universitária junto à comunidade, promovendo desenvolvimento social, com respeito à cultura local, valorizando o conhecimento prévio e oferecendo o conhecimento científico a partir da extensão.

Sendo assim, os profissionais da saúde em formação, aqui destacando a Enfermagem, precisam estar abertos ao uso de novas ferramentas facilitadoras da educação em saúde, aqui destacando o período puerperal, com o uso de Material Educativo, jogos diversos, vídeos, entre outros. Conclui-se que o material educativo proposto no referido projeto foi uma ferramenta eficaz no processo ensino (enfermeiro) e aprendizagem (puérperas), a partir da educação em saúde desenvolvida sobre o pós-parto e aleitamento.

No entanto, novos estudos precisam ser realizados, com outros instrumentos facilitadores do processo de ensino-aprendizagem na prática da educação em saúde, visando identificar as potencialidades e limitações, para que os profissionais, com destaque aos enfermeiros, possam exercer seu papel de educadores para a saúde da população.

\section{Referências}

ABREU, T.F.K.; AMENDOLA, F.; TROVO, M.M. Tecnologias Relacionais como Instrumentos para o Cuidado na Estratégia Saúde da Família. Revista Brasileira de Enfermagem [internet], v.70, n.5, p.1032-9, 2017.

BRASIL. Ministério da Saúde - Instituto Sírio Libanês de Ensino e Pesquisa. Protocolos da Atenção Básica - Saúde das Mulheres. Brasília - DF, 2016. Disponível em: < http://bvsms.saude.gov.br/bvs/publicacoes/protocolos_atencao_basica_saude_mulheres.p df >. Acessado em: 24 abr. 2019. 
CRESTANI, A.H.; MORAES, A.B.; SOUZA, A.P.R. Validação de conteúdo: clareza/pertinência, fidedignidade e consistência interna de sinais enunciativos de aquisição da linguagem. CoDAS, v.29, n.4, e2016180, 2017.

DALMOLIN, A.; PERLINI, N.M.O.G.; COPPETTI, L.C.; ROSSATO, G.C.; GOMES, J.S.; SILVA, M.E.N. Vídeo educativo como recurso para educação em saúde a pessoas com colostomia e familiares. Revista Gaúcha Enfermagem, v.37(esp):e68373, 2016.

D’AVILA, C.G.; PUGGINA, A.C.; FERNANDES, R.A.Q. Construção e validação de jogo educativo para gestantes. Escola Anna Nery, v.22, n.3, 2018.

DODOU, H.D.; OLIVEIRA, T.D.A.; ORIÁ, M.O.B.; RODRIGUES, D.P.; PINHEIRO, P.N.C.; LUNA, I.T. A prática educativa realizada pela enfermagem no puerpério: representações sociais de puérperas. Revista Brasileira Enfermagem, v.70, n.6,p. 1320 8, nov-dez. 2017.

FARIAS, M.S.; PONTE, K.M.A.; GOMES, D.F.; MENEZES, R.S.P. Tecnologia educativa sobre câncer gástrico. Revista Enfermagem UFPE, Recife, v.12, n.4, p.94752, abr. 2018.

FIGUEIREDO, J. V.; FIALHO, A.V.D.; MENDONÇA, G.M.M.; RODRIGUES, D.P.; SILVA, L.F. A dor no puerpério imediato: contribuição do cuidado de enfermagem. Universidade Estadual do Ceará. Revista Brasileira de Enfermagem, Fortaleza, v. 71, suppl 3, p.1424-31, 2018.

LIMA, P.S.; BLANES, L.; FERREIRA, L.M.; GOMES, H.F.C. Manual educativo de cuidados à criança com gastrostomia: construção e validação. REME. Rev Min Enferm., v.22, e-1123, 2018.

MARINHO, M.S.; ANDRADE, E.N.; ABRÃO, A.C.F.V. A atuação do(a) enfermeiro(a) na promoção, incentivo e apoio ao aleitamento materno. Revista Enfermagem Contemporânea, v.4, n2, p.189-198, jul./dez. 2015.

MATOS, M.R.; RAVELLI, A.P.X.; SCORUPSKI, R.; SKUPIEN, S.V.; CAMARGO, T.V. Construção e implementação de um jogo educativo para puérperas. Revista Extensão em Foco, n. 18, p. 01-14, jan./jun. 2019.

MENDES, P.D.G.; FILHA, F.S.C.; SILVA, R.N.A.; VILANOVA, J.M.; SILVA, F.L. O papel educativo e assistencial de enfermeiros durante o ciclo gravídico-puerperal: a percepção de puérperas. R. Interd., v.9, n.3, p.49-56, jul./ago./set. 2016.

NASCIMENTO, E.A.; TARCIA, R.M.L.; MAGALHÃES, L.P.; SOARES, M.A.L.; SURIANO, M.L.F.; DOMENICO, E.B.L. Folhetos educativos em saúde: estudo de recepção. Rev Esc Enferm USP, v.49, n.3, p.435-442, 2015.

OLIVEIRA, J.S.B.; SUTO, C.S.S.; SILVA, R.S. Tecnologias Leves como Práticas de Enfermagem na Atenção Básica. Revista Saúde.Com, v.12, n.2, p.613-621, 2016.

PEUKER, A.C.; LIMA, N.B.; FREIRE, K.M.; OLIVEIRA, C.M.M.; CASTRO, E.K. Construção de um material educativo para a prevenção do câncer de colo do útero.

Estudos Interdisciplinares em Psicologia, Londrina, v. 8, n. 2, p. 146-160, dez. 2017.

PRIGOL, A.P.; BARUFFI, L.M. O Papel do Enfermeiro no Cuidado à Puérpera. Revista de Enfermagem UFSM, v.7, n.1, p.1-8, jan./fev. 2017. 
REGRA, G.L.; SALERNO, G.R.; FERNANDES, S.M.S. Educação em Saúde para Grávidas e Puérperas. Revista Pesquisa em Fisioterapia, v.7, n.3, p.351-358, ago. 2017.

SABINO, L.M.M.; BRASIL, D.R.M.; CAETANO, J.A.; SANTOS, M.C.L.; ALVES, M.D.S. Uso de Tecnologia Leve-Dura nas Práticas de Enfermagem: Análise de Conceito. Chía, Colombia, v.16, n.2, p.230-239, jun. 2016. 